\title{
Contrast Agent Name
}

National Cancer Institute

\section{Source}

National Cancer Institute. Contrast Agent Name. NCI Thesaurus. Code C69294.

The identity of the contrast agent. 